\title{
Tratamento da UCE refratária aos anti-histamínicos e na impossibilidade do omalizumabe, nos adultos
}

\author{
Treatment of CSU refractory to antihistamines and \\ in the impossibility of omalizumab therapy in adults \\ Bruna Gehlen1, Mariana Mousinho-Fernandes', Paula Natassya Argolo1, \\ Grazielly de Fátima Pereira ${ }^{1}$, Jorge Kaliil ${ }^{1}$, Antônio Abilio Motta ${ }^{1}$, Rosana Câmara Agondi ${ }^{1}$
}

\section{RESUMO}

Há o empenho contínuo de especialistas no desenvolvimento de tratamentos resolutivos ou eficazes nos controles das doenças, no entanto, a entidade urticária crônica espontânea (UCE), quando refratária à primeira linha de tratamento, os anti-histamínicos, apresenta um prognóstico desfavorável. Existe um arsenal de medicamentos biológicos disponíveis já consolidados como eficazes e seguros, porém eventualmente nos defrontamos com a inacessibilidade a estes medicamentos, devido aos custos dos mesmos e aos trâmites necessários para dar início ao tratamento. Tais fatos fundamentam a discussão sobre terapias alternativas com outros fármacos, visando manter o manejo adequado da doença e a qualidade de vida dos pacientes.

Descritores: Urticária, imunossupressores, ciclosporina.

\section{Introdução}

Urticária crônica espontânea (UCE) é caracterizada pelo desenvolvimento de urticas, angioedema ou ambos, com duração de pelo menos 6 semanas. $\mathrm{Na}$ UCE, não se identificam agentes externos ou fatores específicos na sua etiologia, e a exclusão de diagnósticos diferenciais se faz necessária ${ }^{1,2}$.

A fisiopatologia da UCE não está totalmente esclarecida, porém, a ativação dos mastócitos cutâneos e a liberação de seus mediadores inflamatórios são a via final comum de todas as formas de urticária. Estudos sustentam esta hipótese pela demonstração

\section{ABSTRACT}

Specialists have made a continuous effort for the development of effective treatments for disease control; however, chronic spontaneous urticaria (CSU), when refractory to the first line of treatment, ie, antihistamines, has an unfavorable prognosis. There are biological medicines available, which have been consolidated as effective and safe, but we are occasionally faced with a lack of access to these medicines due to their costs and the necessary procedures to start treatment. Such facts support the discussion about alternative therapies with other drugs, aiming at maintaining the adequate management of the disease and the quality of life of patients.

Keywords: Urticaria, immunosuppressive agents, cyclosporine.

de concentrações aumentadas de histamina no tecido cutâneo e pela resposta clínica da urticária aos antihistamínicos ${ }^{3}$.

Portanto, medicamentos anti-histamínicos e aqueles que impeçam a desgranulação dos mastócitos/ basófilos são os medicamentos com maior potencial para o controle da UCE. Os anti-histamínicos são agonistas inversos dos receptores $\mathrm{H} 1$ da histamina, ou seja, a histamina se liga a sítios específicos no receptor $\mathrm{H} 1$ levando à ativação do receptor; os antihistamínicos se ligam a outros sítios no receptor $\mathrm{H} 1$,

1. Hospital das Clínicas da Faculdade de Medicina da Universidade de São Paulo, Serviço de Imunologia Clínica e Alergia - São Paulo, SP, Brasil. 
inativando este receptor. Os anti-histamínicos de primeira geração, mais antigos, incluem clorfeniramina, difenidramina, hidroxizina e cetotifeno, penetram prontamente no sistema nervoso central, onde causam sedação, sonolência, fadiga e prejudicam a concentração e a memória; causam diminuição do desempenho cognitivo e psicomotor. O uso de anti-histamínicos de primeira geração deve ser desencorajado e não é recomendado pelo consenso mundial de manejo e tratamento da urticária crônica ${ }^{1,4,5}$.

Os anti-histamínicos $\mathrm{H} 1$ de segunda geração, mais novos, são mais seguros, causam menos sedação e são mais eficazes. Destes, azelastina, olopatadina, cetirizina e loratadina podem causar sonolência em alguns indivíduos, mas isso geralmente é leve. Publicações recentes sugeriram que, em doses recomendadas pelo fabricante, a levocetirizina é menos sedativa do que cetirizina. A ocupação dos receptores de histamina no cérebro pelas fexofenadina e bilastina é desprezível. Isto ocorre devido ao mecanismo de ação destes dois anti-histamínicos, eles são substratos para a glicoproteína-P, que é uma proteína transportadora transmembrana, uma bomba de efluxo dependente de ATP que limita a passagem destes medicamentos através da barreira hematoencefálica. Os anti-histamínicos de segundageração têm um início de ação rápido entre 1 e 4 horas, e são clinicamente eficazes por 24 horas $^{5}$.
Portanto, o tratamento de primeira linha da UCE, conforme a diretriz mundial, é o anti-histamínico $\mathrm{H} 1$ de segunda geração, porém, a terapia com anti-histamínicos $\mathrm{H} 1$ leva à ausência de sintomas em menos de $50 \%$ dos pacientes com UCE, mesmo esses sendo eficazes na redução do prurido, eritema, número, tamanho e duração da urticária ${ }^{6,7}$.

$\mathrm{Na}$ urticária, devido aos altos níveis locais de histamina na pele, as doses padronizadas de antihistamínicos frequentemente não são capazes de controlar os sintomas. Nesse caso, se os sintomas persistirem após duas semanas, a segunda linha de tratamento da UCE é aumentar a dosagem de um mesmo anti-H1 de segunda geração em até quatro vezes ${ }^{5}$.

Por fim, vários estudos apoiam a segurança em utilizar-se anti-histamínicos $\mathrm{H} 1$, em doses quadruplicadas ou administradas prolongadamente nos pacientes com UCE ${ }^{1,6,7}$. Deve-se orientar o paciente quanto à aceitabilidade do medicamento e à adesão ao tratamento, visto que isso afeta os resultados. É importante escolher os anti-histamínicos de modo individualizado a cada paciente, visando controle de sintomas e buscando menos efeitos colaterais, como dificuldade na performance cognitiva e rendimento, além de prejuízo sobre a qualidade de vida dos pacientes, desestimulando os mesmos a seguir 0 tratamento indicado 8 .

Tabela 1

Anti-histamínicos e suas particularidades

\begin{tabular}{lcc}
\hline Características & AH1 de $1^{\text {a }}$ geração & AH1 de ${ }^{\mathrm{a}}$ geração \\
\hline $\begin{array}{l}\text { Início de ação } \\
\text { Duração de ação }\end{array}$ & Menor lento (múltiplas doses) & Mais rápido \\
Seletividade pelo receptor H1 & Baixa & Alta \\
Efeitos colaterias & Sonolência, alteração cognitiva, \\
efeito anticolinérgico, metabolização hepática & Pouco frequentes
\end{tabular}


Para os pacientes refratários aos anti-histamínicos, conforme a diretriz mundial de urticária, a indicação é adicionar-se o omalizumabe ao anti-histamínico. $O$ omalizumabe é um anticorpo monoclonal humanizado IgG1, anti-lgE. Ele se liga ao domínio C\&3 da IgE livre formando complexos inativos, o domínio C\&3 é o local de ligação da IgE com seus receptores de alta afinidade nos mastócitos e basófilos, portanto, o omalizumabe impede a ligação das moléculas de lgE livres com seus receptores. Com a redução das moléculas de lgE ligadas aos seus receptores nos mastócitos e basófilos, há internalização destes receptores e não há a desgranulação celular 1,5,9.

O omalizumabe e os anti-histamínicos em dose indicada em bula são os únicos medicamentos aprovados para o tratamento da UCE, sendo que este último é efetivo e seguro nas doses de 150 e $300 \mathrm{mg}$ ao mês ${ }^{1,10-15}$.

Zhao ZT e cols. ${ }^{16}$, em uma metanálise de estudos clínicos randomizados, mostraram que o omalizumabe foi mais eficaz que o placebo no tratamento de pacientes com urticária crônica espontânea e também apresentou um perfil de segurança semelhante ao placebo. A resposta ao algoritmo de tratamento da UCE, recomendado pelo consenso mundial, está em torno de $55 \%$ para os anti-histamínicos (uma a quatro vezes ao dia), e entre $65 \%$ e $80 \%$ para o omalizumabe. Quando o paciente não responde à adição de omalizumabe ao anti-histamínico, a opção é a substituição do omalizumabe pela ciclosporina, sendo que até $70 \%$ dos pacientes podem alcançar o controle da UCE com este medicamento; entretanto, a frequência de eventos colaterais é elevada ${ }^{17}$.

\section{Tratamento da UCE refratária aos anti- histamínicos e inacessibilidade ao omalizumabe}

De acordo com a diretriz mundial de urticária, o objetivo do tratamento da UCE é alcançar o controle completo dos sintomas, até que a doença entre em remissão (the goal of treatment is to treat the disease until it is gone), considerando-se priorizar os medicamentos com melhor eficácia e maior segurança para o paciente ${ }^{1}$. O algoritmo de tratamento da UCE conforme consenso mundial está demonstrado na Figura 1.

\section{Diretrizes EAACI/GA2LEN/EDF/WAO}

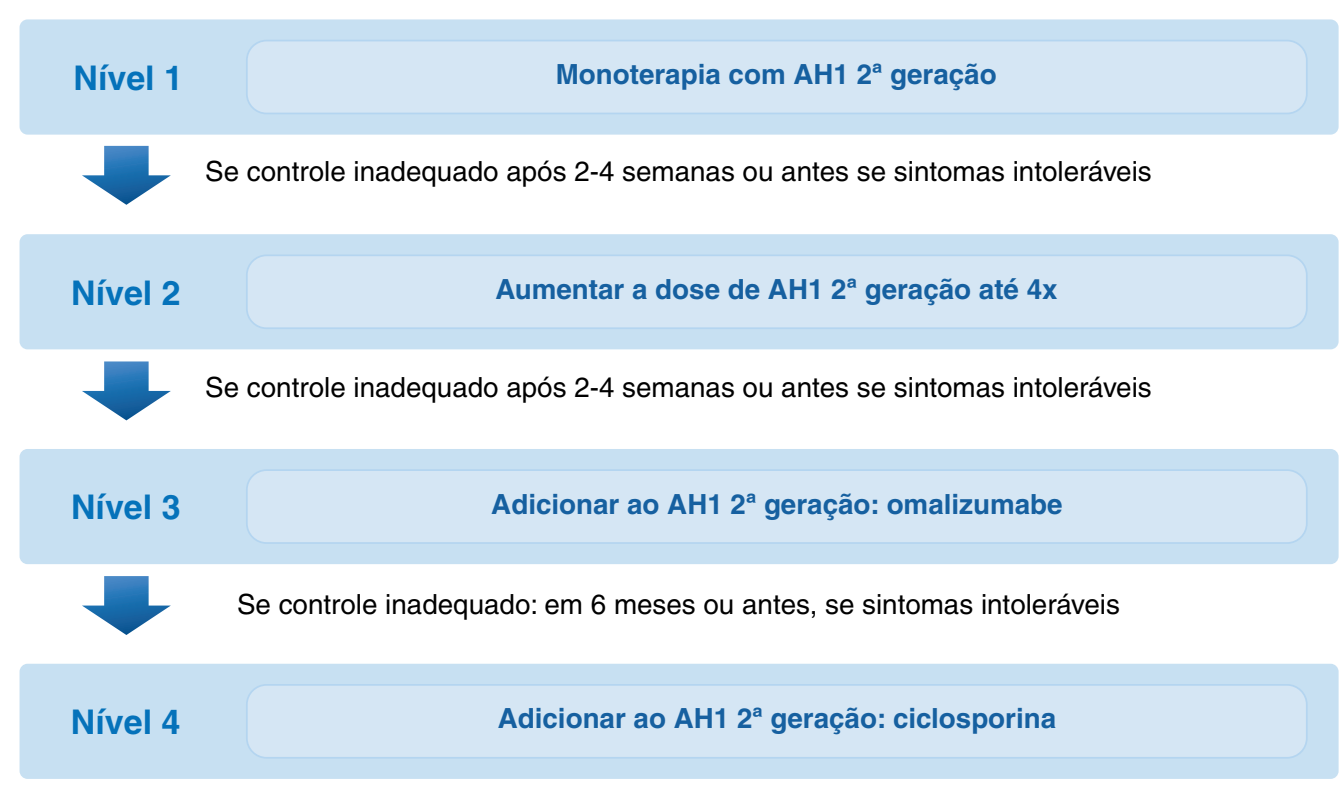

\section{Figura 1}

Algoritmo de tratamento da urticária crônica espontânea recomendado por consenso mundial Modificado de Zuberbier et al. ${ }^{1}$. 
Existem questionários para a avaliação inicial da doença e que devem ser utilizados para o acompanhamento do paciente após o início do tratamento. São ferramentas de avaliação validadas para o seguimento do paciente: o Escore de atividade da urticária (UAS), o Escore de atividade do angioedema (AAS), - Questionário de qualidade de vida da urticária crônica (CU-Q2oL), o Questionário de qualidade de vida do angioedema (AE-QoL) e o Teste de controle da urticária (UCT). Logo, as pontuações nesses questionários podem ser usadas para orientar a seleção do tratamento de acordo com o status da doença do paciente ${ }^{1,18}$. Kaplan e cols. ${ }^{19}$ sugeriram que a resposta ao tratamento poderia ser baseada no UAS7 (UAS usado pelo período de 7 dias, com escore variando de 0 a 42), sendo a resposta completa quando UAS7 $=0$ e urticária bem controlada quando UAS7 $\leq 6$.

$O$ algoritmo mundial de tratamento da UCE visa utilizar os medicamentos mais eficazes e seguros, entretanto, vários pacientes não respondem aos anti-histamínicos, e alguns outros também não respondem ao omalizumabe ou não têm acesso a este medicamento. Nesta situação, podemos fazer uso de medicamentos alternativos, devendo-se considerar a eficácia, a segurança, a conveniência e o custo destes medicamentos ${ }^{20}$. Vários medicamentos alternativos têm sido utilizados para o manejo de pacientes com UCE refratária. As evidências que sustentam a eficácia para a maioria destes medicamentos são limitadas e, apesar disso, o uso de medicamentos alternativos tem o objetivo de melhorar a qualidade de vida e reduzir o uso crônico de corticoide sistêmico ${ }^{21}$.

Vários medicamentos foram sugeridos na literatura como opções terapêuticas alternativas para UCE, entretanto, as evidências de eficácia são baixas. Embora a manutenção destes medicamentos alternativos em um algoritmo de tratamento não seja recomendada, eles podem ter relevância clínica, pois são acessíveis em alguns sistemas de saúde. Alguns medicamentos podem ser eficazes para certos grupos de pacientes. A escolha do medicamento alternativo dependerá da experiência clínica individual do médico ${ }^{1,22}$.

\section{Ciclosporina}

A ciclosporina é recomendada como quarta linha de tratamento da UCE, conforme as orientações de diretrizes nacionais e internacionais. A ciclosporina é um medicamento imunossupressor potente que inibe a ativação do linfócito $T$ bloqueando a produção de citocinas inflamatórias. A ciclosporina foi o primeiro medicamento imunossupressor descrito que agia seletivamente nos linfócitos, especialmente os linfócitos $\mathrm{T}$ helper. $\mathrm{O}$ complexo entre a ciclosporina e o seu receptor citoplasmático, a ciclofilina, inibe a atividade da fosfatase de calcineurina, uma fosfatase dependente de calmodulina, resultando na diminuição da transcrição de genes de citocinas (IL-2, 3, 4 e TNF-alfa). Além da inibição de linfócito T, estudos in vitro mostraram que leucócitos pré-incubados com ciclosporina inibiam a liberação de histamina após estímulo com soro de pacientes com UCE. Estudos também mostraram que a ciclosporina inibiu a liberação de histamina mediada por IgE dos mastócitos por um efeito dependente da sua concentração e, também, que após o uso de ciclosporina houve uma redução na atividade de liberação de histamina sérica e na resposta do teste do soro autólogo (TSA). Esses achados sustentam a hipótese de que os mecanismos de ação da ciclosporina, na UCE, podem envolver efeitos nas células $T$ ativadas, nos basófilos, e, também, nos mastócitos ${ }^{1,23,24}$.

Alguns fatores estariam associados a uma melhor resposta à ciclosporina. Grattan e cols. ${ }^{23}$ mostraram que dois terços dos pacientes com UCE, com resposta incompleta ao anti-histamínico e teste do soro autólogo positivo, apresentaram melhora clínica após o uso da ciclosporina. Um estudo de metanálise mostrou que a taxa de resposta à ciclosporina dos pacientes com UCE pode chegar a $70 \%{ }^{25}$.

$\mathrm{Na}$ UCE, estudos mostraram que a dose entre 2,5 a $3 \mathrm{mg} / \mathrm{kg} / \mathrm{dia}$ foi considerada eficaz por 4 a 6 semanas, mostrando melhora significante no controle da doença. $O$ tratamento com ciclosporina em baixa dose $(2,5 \mathrm{mg} / \mathrm{kg} / \mathrm{dia})$ reduziu os níveis séricos de citocinas IL-2, IL-5 e fator de necrose tumoral alfa, que se encontram aumentados nos pacientes com UCE. O tempo exato de duração do tratamento com a ciclosporina não foi definido, a maioria necessitaria deste medicamento por 3 meses, enquanto alguns pacientes utilizariam o medicamento por 2 a 3 anos. O uso de longo prazo, no entanto, não é recomendado. A dose de ciclosporina sugerida por alguns autores é de $3 \mathrm{mg} / \mathrm{kg} / \mathrm{d}$ (dividida em duas doses) por 6 semanas, seguida de redução gradual por mais 6 semanas 24,26 .

Vários efeitos colaterais estão associados ao uso da ciclosporina, especialmente com doses mais elevadas. Um dos efeitos colaterais mais comuns é a toxicidade renal, que se apresenta com aumento da creatinina sérica. $\mathrm{O}$ quadro pode se apresentar como nefrotoxicidade aguda, reversível e dependente da 
dose, que é secundária à vasoconstrição das arteríolas aferentes e disfunção dos túbulos. Entretanto, em alguns casos, a nefrotoxicidade também pode se apresentar com dano renal progressivo, crônico e irreversível que resulta da toxicidade renal cumulativa em pacientes com tratamento de longo prazo. O quadro deve ser acompanhado rigorosamente, podendo-se, eventualmente reduzir a dose, mas sugere-se suspender este medicamento 26 .

Se houver uma elevação da creatinina sérica acima de $25 \%$ a $30 \%$ do basal em duas leituras separadas, realizadas com 2 semanas de intervalo, a dose da ciclosporina deve ser reduzida em $25 \%$ e a creatinina deve ser verificada novamente em 1 a 2 semanas. Além do controle da creatinina sérica, deve-se acompanhar os valores séricos de potássio, bilirrubina, ácido úrico, magnésio e enzimas hepáticas. $O$ controle pressórico também deve ser monitorado 26,27 .

A hipertensão induzida pela ciclosporina se desenvolve durante os estágios iniciais, nas primeiras semanas de tratamento. Isso ocorre devido à vasoconstricção renal e retenção de sódio, que é dosedependente e responde bem à redução da dose de ciclosporina ${ }^{26}$.

\section{Opções terapêuticas alternativas para tratamento da UCE}

\section{Montelucaste}

Os cisleucotrienos são mediadores pró-inflamatórios potentes cujos efeitos podem ser bloqueados pelos antagonistas dos receptores de leucotrienos, como o montelucaste, zafirlucaste e pranlucaste. Vários estudos avaliaram o efeito destes medicamentos no tratamento da UCE, porém, esta eficácia é controversa na literatura, resultando em uma não recomendação deste medicamento no algoritmo de tratamento da UCE pelas diretrizes nacionais e internacionais. Entretanto, alguns estudos mostraram uma boa eficácia para os pacientes com UCE exacerbada por anti-inflamatórios não esteroidais (AINEs). O montelucaste inibe o receptor de leucotrieno D4 (LTD4), e alguns estudos mostraram redução do UAS7 nos pacientes com UCE. O montelucaste não deve ser usado em monoterapia ${ }^{20,28}$.

Sarkar TK e cols. ${ }^{29}$, em um estudo duplo-cego randomizado, compararam o uso da levocetirizina $10 \mathrm{mg} /$ dia isolada à levocetirizina $5 \mathrm{mg} /$ dia associada ao montelucaste $10 \mathrm{mg} / \mathrm{dia}$. Os autores concluíram que ambos os tratamentos melhoram escores de atividade da doença, entretanto, os efeitos colaterais dos anti-histamínicos, como a sonolência, foram mais frequentes nos pacientes que utilizaram levocetirizina $10 \mathrm{mg} / \mathrm{dia}$.

Recentemente, Akenroye AT e cols. avaliaram retrospectivamente o uso do montelucaste associado ao anti-histamínico como segunda linha de tratamento para pacientes com UCE nos quais o angioedema era a manifestação predominante. Os autores observaram que houve uma melhora significante da gravidade e da frequência da doença em mais de $90 \%$ dos pacientes. Entretanto, este estudo apresenta limitações, servindo como incentivo a futuros estudos ${ }^{30}$.

Vários estudos na literatura descreveram a segurança do montelucaste, entretanto, outros mais recentes relataram reações adversas graves ao montelucaste que compreendem as reações alérgicas e dor torácica. Há uma associação entre o montelucaste e a granulomatose eosinofílica com poliangiite não bem esclarecida, entretanto, deve-se ficar atento às manifestações clínicas desta enfermidade rara. Adultos e crianças podem evoluir com alterações neuropsiquiátricas ao se iniciar o tratamento com o montelucaste. Os eventos adversos descritos na literatura, em crianças e adultos, incluem angioedema, depressão, agressividade, cefaleia, pesadelos e ideação suicida ${ }^{31}$.

Conforme diretrizes mundiais, o nível de evidência que sustenta a utilização do antagonista de receptor de leucotrieno é baixo. Devido ao seu custo e a uma boa tolerância, este medicamento foi recomendado como tratamento complementar em caso de indisponibilidade do omalizumabe, porém, as diretrizes mundiais de manejo e tratamento da urticária crônica não incluíram o montelucaste no algoritmo de tratamento da UC, sendo uma opção terapêutica conforme a experiência do especialista. Entretanto, no momento, seu uso não está aprovado para o tratamento de $\mathrm{UCE}^{6}$.

\section{Hidroxicloroquina}

A hidroxicloroquina é um medicamento antimalárico frequentemente usado para reduzir a inflamação nas condições autoimunes e inflamatórias. Em adultos, a hidroxicloroquina pode ser usada como agente alternativo para tratar UCE refratária aos anti-histamínicos. Estudos observaram que a hidroxicloroquina foi eficaz, a curto prazo, como opção 
terapêutica adjuvante alternativa no tratamento de UCE refratária aos anti-histamínicos em adultos, contribuindo para a remissão da doença ${ }^{32,33}$. Entretanto, o mecanismo de ação da hidroxicloroquina no tratamento da UCE é desconhecido, e as descrições de êxito do seu uso no tratamento são limitadas. Alguns estudos relatam como possíveis mecanismos na UCE, a supressão da ativação dos linfócitos $T$ e a interferência na apresentação antigênica e na alcalinização de vacúolos intracelulares ${ }^{33,34}$.

A hidroxicloroquina é considerada um medicamento bem tolerado, administrado por via oral, de baixo custo e com efeitos colaterais graves infrequentes. A dose utilizada de hidroxicloroquina na UCE é de $200 \mathrm{mg}$ duas vezes ao $\mathrm{dia}^{32,33,35}$.

As desvantagens da hidroxicloroquina incluem a necessidade de 2 a 3 meses para se atingir 0 efeito desejado do medicamento, além da necessidade de monitoração oftalmológica previamente e no seguimento do paciente devido ao potencial de retinopatia pelo medicamento. A medicação eleva o risco de retinopatia, condição que pode ser irreversível, após os primeiros 5 anos de uso, logo se preconiza a avaliação oftalmológica no primeiro ano e anualmente após os 5 primeiros anos. Outros efeitos colaterais, que de modo geral são reversíveis, incluem sintomas gastrointestinais, cefaleia e prurido cutâneo 34,35,36.

\section{Dapsona}

A dapsona é um antibiótico sulfonamida que inibe a quimiotaxia de neutrófilos e a produção de espécies reativas de oxigênio; isso explicaria sua eficácia em doenças neutrofílicas, como pioderma gangrenoso e urticária vasculite. A dapsona também inibe a 5-lipooxigenase nas células polimorfonucleares humanas e em mastócitos de camundongos, levando à redução da produção de leucotrienos ${ }^{37}$.

O mecanismo de ação na UCE não foi totalmente esclarecido, entretanto, os efeitos anti-inflamatórios da dapsona abrangem uma variedade de possíveis propriedades relevantes na urticária. Estas incluem interferência com a liberação ou função de enzimas lisossomais e com a geração de mieloperoxidase, mas, também interferência com a adesividade de neutrófilos mediada por integrina e inibição da atividade de prostaglandinas e leucotrienos ${ }^{36,38,39}$.

As doses variam de 50 a $100 \mathrm{mg}$ diários, e para os respondedores, a eficácia foi aparente após uma semana. O principal evento adverso com a dapsona é a anemia, em média $80 \%$ dos pacientes em uso de 100-150 mg/dia apresentam uma queda de pelo menos $1 \mathrm{~g} / \mathrm{dL}$ na concentração de hemoglobina, outro efeito colateral comum é a metemoglobinemia, sendo que a maioria dos pacientes apresenta-se assintomático e este efeito se resolve com a suspensão do medicamento. Outros efeitos colaterais menos comuns incluem anemia hemolítica devido à deficiência de G6PD, agranulocitose, neuropatia periférica, cefaleia, nefrite e hipotireoidismo. Todos os pacientes devem realizar previamente avaliação de hemograma completo, de função renal e de G6PD; além disso, os pacientes deveriam ser monitorados mensalmente através de avaliação de hemograma e função renal nos primeiros 6 meses e depois periodicamente ${ }^{35,39}$. No entanto, este medicamento continua não comprovado como tratamento eficaz para a urticária crônica e necessita de mais estudos clínicos que favoreçam o uso dessa terapia em UCE 27,38 .

\section{Sulfassalazina}

Os mecanismos de ação não são bem conhecidos, mas estudos mostraram que a sulfassalazina reduziria a síntese de anticorpos e influenciaria a produção de linfócitos T e citocinas. Alguns estudos mostraram que a sulfassalazina atua na liberação de histamina de mastócitos mediada por lgE, mas esses resultados não foram confirmados por outros pesquisadores. A sulfassalazina também aumenta a produção de prostaglandina E2, que pode ter propriedades anti-inflamatórias, como a inibição da liberação de mediadores inflamatórios dos mastócitos. A sulfassalazina também inibe a síntese de leucotrienos. Em um estudo com pacientes com UCE corticodependente, a introdução da sulfassalazina reduziu ou descontinuou o uso de corticoide ${ }^{37}$.

Estudos sugerem que se inicie a dose de sulfassalazina com $500 \mathrm{mg} / \mathrm{dia}$ e que se aumente $500 \mathrm{mg}$ semanalmente até a dose de $2 \mathrm{~g} / \mathrm{dia}$. Para os respondedores à sulfassalazina, seu efeitos já podem ser observados com 1 mês do início do tratamento. As doses acima de $2 \mathrm{~g} /$ dia não adicionam benefício e estão associadas a efeitos colaterais mais graves. Os efeitos colaterais mais comuns são sintomas gastrointestinais e cefaleia. Outros efeitos colaterais são as alterações hematológicas, especificamente leucopenia. Estas alterações são mais frequentes com doses mais elevadas de sulfassalazina ${ }^{34,36,40}$. 


\section{Tabela 2}

Características gerais de medicamentos alternativos para o tratamento da urticária crônica espontânea

\begin{tabular}{|c|c|c|c|}
\hline Medicamento & Dose preconizada & Início de ação & Principais efeitos colaterais \\
\hline Montelucaste & $10 \mathrm{mg} / \mathrm{dia}$ & 1 semana & $\begin{array}{l}\text { Angioedema, dor torácia, } \\
\text { manifestações neuropsiquiátricas }\end{array}$ \\
\hline Hidroxicloroquina & $400 \mathrm{mg} / \mathrm{dia}$ & 2 a 3 meses & $\begin{array}{l}\text { Sintomas gastrointestinais, leucopenia, } \\
\text { hepatotoxicidade, nefrotoxicidade e retinopatia }\end{array}$ \\
\hline Dapsona & 50 a $100 \mathrm{mg} / \mathrm{dia}$ & 1 semana & $\begin{array}{c}\text { Anemia, metemoglobinemia, } \\
\text { anemia hemolítica quando deficiência de G6PD }\end{array}$ \\
\hline Sulfassalazina & Até $2 \mathrm{~g} / \mathrm{dia}$ & 1 mês & $\begin{array}{l}\text { Sintomas gastrointestinais, cefaleia e } \\
\text { alteracões hematológicas (leucopenia) }\end{array}$ \\
\hline Metotrexato & 7,5 a $15 \mathrm{mg} / \mathrm{kg} / \mathrm{dia}$ & 2 semanas & $\begin{array}{l}\text { Sintomas gastrointestinais, rash, } \\
\text { hepatoxicidade, infecções }\end{array}$ \\
\hline Colchicina & $0,6 \mathrm{mg} 2$ vezes ao dia & 1 mês & $\begin{array}{c}\text { Sintomas gastrointestinais, depressão, rash, } \\
\text { neuropatia perférica }\end{array}$ \\
\hline
\end{tabular}

Fonte: Khan ${ }^{21}$, Seth $^{34}$ e Morgan ${ }^{36,39}$.

G6PD = glicose-6-fosfato desidrogenase.

\section{Outras opções}

O conhecimento atual sobre a complexidade da fisiopatologia da UCE onde outros mediadores e células, além dos mastócitos, basófilos e histamina, participam, nos fornece outros alvos potenciais para o tratamento da UCE. Os medicamentos preconizados para o tratamento de doenças autoimunes e inflamatórias demonstraram eficácia no tratamento da UCE e, portanto, são potenciais alternativas terapêuticas para pacientes que não respondam aos anti-histamínicos. Na ausência de acesso ou de eficácia do omalizumabe, ou ainda no aguardo de outras opções de medicamentos biológicos para tratamento da UCE, outros medicamentos anti-inflamatórios ou imunossupressores poderiam ser utilizados de forma individualizada, além dos descritos acima ${ }^{37}$.

Azatioprina, metotrexato, interferon, plasmaférese, fototerapia, imunoglobulina intravenosa e outras opções de tratamento têm pouca evidência, ou apenas foram relatadas. Apesar da falta de evidência em publicações, todos esses medicamentos podem ser úteis para pacientes em contexto clínico individualizado ${ }^{1}$.

\section{Considerações finais}

Devido ao papel central dos mastócitos, basófilos e da histamina na fisiopatologia da UCE, os anti-histamínicos continuam sendo o tratamento de primeira linha. No entanto, o conhecimento atual sobre essa doença complexa também reconhece um papel importante dos linfócitos T, linfócitos B, eosinófilos e autoanticorpos. As implicações desses outros mediadores fornecem, portanto, outros alvos para o tratamento da UCE. Os medicamentos preconizados para o tratamento de doenças autoimunes e inflamatórias demonstraram eficácia no tratamento da UCE e, portanto, são potenciais alternativas terapêuticas para pacientes que não respondam aos anti-histamínicos. $\mathrm{Na}$ ausência de acesso ou de eficácia às outras linhas de tratamento para UCE recomendadas pelas diretrizes mundiais, outros medicamentos anti-inflamatórios ou imunossupressores poderiam ser utilizados de forma individualizada.

Porém, antes de indicar qualquer uma das alternativas, devemos sempre levar em consideração as comorbidades de cada paciente; as contraindicações, tempo de ação, efeitos colaterais dos medicamentos, 


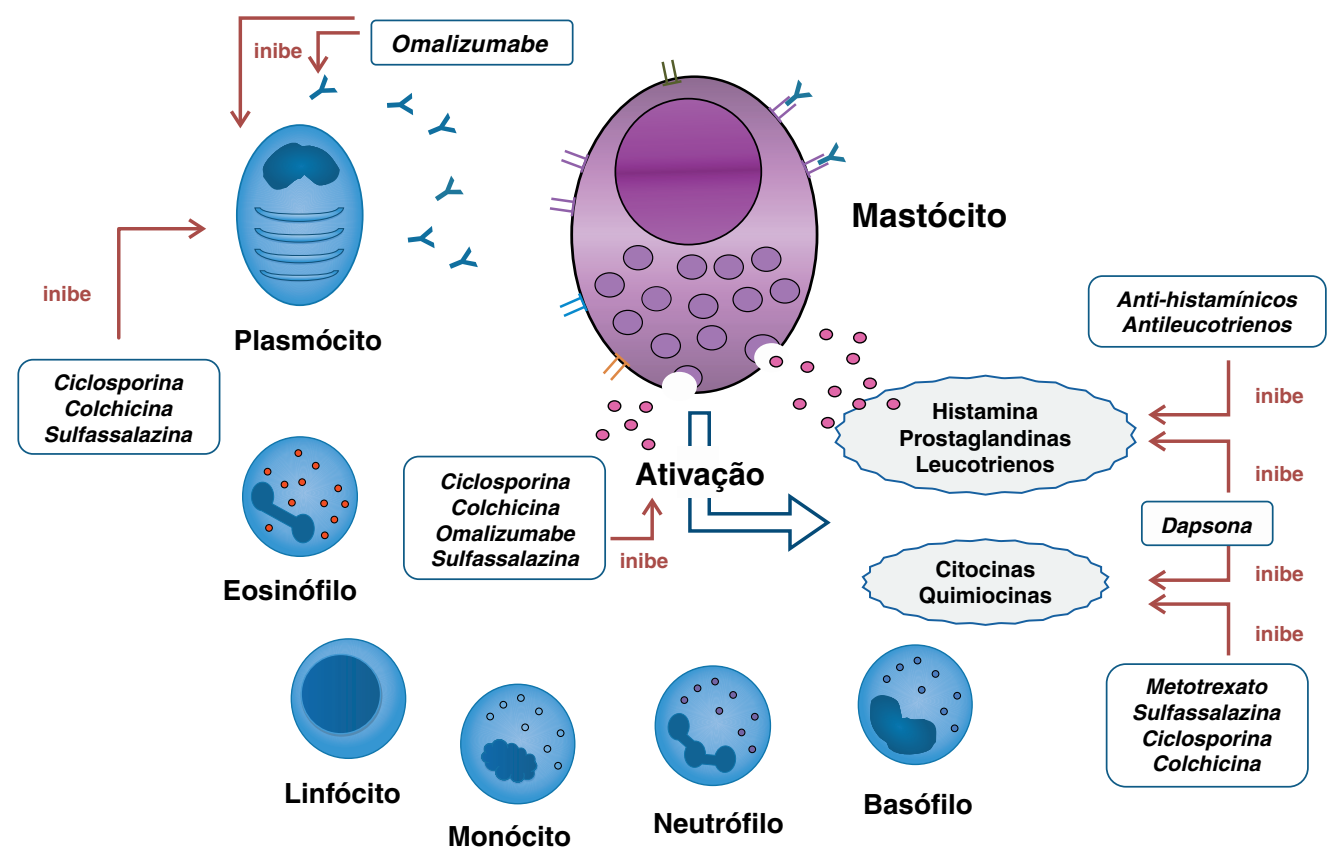

\section{Figura 2}

Prováveis alvos terapêuticos na UCE e mecanismos de ação de diversos medicamentos descritos na literatura

Modificado de de Montojoye $\mathrm{L}^{37}$.

assim como a experiência do médico, custo e disponibilidade da medicação e preferência do paciente. Além disso, são necessários novos estudos para padronizar a indicação precisa de cada medicação.

\section{Referências}

1. Zuberbier T, Aberer W, Asero R, Abdul Latiff AH, Baker D, BallmerWeber $\mathrm{B}$, et al. The EAACI/GA2LEN/EDF/WAO guideline for the definition, classification, diagnosis and management of urticaria. Allergy. 2018;73:1393-414.

2. Maurer M, Church MK, Marsland AM, Sussman G, Siebenhaar F, Vestergaard FC, et al. Questions and answers in chronic urticaria: where do we stand and where do we go? J Eur Acad Dermatol Venereol. 2016;30(Suppl 5):7-15.

3. Bracken SJ, Abraham S, MacLeod AS. Autoimmune theories of chronic spontaneous urticaria. Front Immunol. 2019;10:627.

4. Church MK, Maurer M, Simons FER, Bindslev-Jensen $C$, van Cauwenberge $P$, Bousquet $J$, et al. Risk of firstgeneration $\mathrm{H} 1$-antihistamines: a GA2LEN position paper. Allergy. 2010;65:459-66.

5. Church MK.Does antihistamine up-dosing solve chronic spontaneous urticaria? Curr Treat Options Allergy. 2016;3:416-22.

6. Vestergaard C, Toubi E, Maurer M, Triggiani M, Ballmer-Weber B, Marsland $\mathrm{A}$, et al. Treatment of chronic spontaneous urticaria with an inadequate response to $\mathrm{H} 1$-antihistamines: an expert opinion. Eur J Dermatol. 2017;27(1):10-19.
7. Parisi GF, Leonardi S, Ciprandi G, Corsico A, Licari A, del Giudice $\mathrm{MM}$, et al. Cetirizine use in childhood: an update of a friendly 30 -year drug. Clin Mol Allergy. 2020;18:2.

8. Baharudin A, Abdul Latiff AH, Woo K, Yap FBB, Tang IP, Leong KF, et al. Using patient profiles to guide the choice of antihistamines in the primary care setting in Malaysia: expert consensus and recommendations. Ther Clin Risk Manag. 2019;15:1267-75.

9. Gasser P, Tarchevskaya SS, Guntern P, Brigger D, Ruppli R, Zbären $\mathrm{N}$, et al. The mechanistic and functional profile of the therapeutic anti-IgE antibody ligelizumab differs from omalizumab. Nat Commun. 2020;11:165.

10. Kaplan A, Ledford D, Ashby M, Canvin J, Zazzali JL, Conner E, et al. Omalizumab in patients with symptomatic chronic idiopathic/ spontaneous urticaria despite standard combination therapy. $J$ Allergy Clin Immunol. 2013;132:101-9.

11. Saini SS, Bindslev-Jensen C, Maurer M, Grob JJ, Baskan EB, Bradley MS, et al. Efficacy and safety of omalizumab in patients with chronic idiopathic/spontaneous urticaria who remain symptomatic on $\mathrm{H} 1$ antihistamines: a randomized, placebo-controlled study. J Invest Dermatol. 2015;135:67-75

12. Maurer M, Vena GA, Cassano N, Zuberbier T. Current and future therapies for treating chronic spontaneous urticaria. Expert Opin Pharmacother. 2016;17:1131-9.

13. Deza G, Ricketti PA, Giménez-Arnau AM, Casale TB. Emerging biomarkers and therapeutic pipelines for chronic spontaneous urticaria. J Allergy Clin Immunol Pract. 2018;6:1108-17.

14. Incorvaia C, Mauro M, Makri E, Leo G, Ridolo E. Two decades with omalizumabe: what we still have to learn. Biologics: Targets and Therapy. 2018;12:135-42. 
15. Francés $L$, Leiva-Salinas $M$, Silvestre JF. Omalizumabe in the treatment of chronic urticaria. Actas Dermosifiliogr. 2014;105:45-52.

16. Zhao ZT, Ji CM, Meng L, Hawro T, Wei JF, Maurer M. Omalizumab for the treatment of chronic spontaneous urticaria: a metaanalysis of randomized clinical trials. J Allergy Clin Immunol. 2016;137:1742-50.

17. Kaplan AP. Treatment of urticaria: a clinical and mechanistic approach. Curr Opin Allergy Clin Immunol. 2019;19:387-92.

18. Giménez Arnau AM, Santiago AV, Tomás JB, Presa IJ, Horrillo ML, Miguel FJM, et al. Therapeutic strategy according to the differences in response to omalizumab in patients with chronic spontaneous urticaria. J Investig Allergol Clin Immunol. 2019;29:338-48.

19. Kaplan A, Ferrer M, Bernstein JA, Antonova E, Trzaskoma B, Raimundo $\mathrm{K}$, et al. Timing and duration of omalizumab response in patients with chronic idiopathic/spontaneous urticaria. J Allergy Clin Immunol. 2016;137:474-81.

20. Holm JG, Ivyanskiy I, Thomsen SF. Use of nonbiologic treatments in antihistamine refractory chronic urticaria: a review of published evidence. J Dermatol Treat. 2018;29:80-97.

21. Khan DA. Alternative agents in refractory chronic urticaria: evidence and considerations on their selection and use.J Allergy Clin Immunol Pract. 2013;1:433-40.

22. Vena GA, Maurer M, Cassano N, ZuberbierT. Alternative treatments for chronic spontaneous urticaria beyond the guideline algorithm. Curr Opin Allergy Clin Immunol. 2017;17:278-85.

23. Grattan CEH, O'Donnell BFO, Francis DM, Niimi N, Barlow RJ, Seed PT, et al. Randomized double-blind study of cyclosporin in chronic 'idiopathic' urticaria. Br J Dermatol. 2000;143:365-72.

24. Amor KT, Ryan C, Menter A.The use of cyclosporine in dermatology: part I. J Am Acad Dermatol. 2010;63:925-46.

25. Kulthanan K, Subchookul C, Hunnangkul S, Chularojanamontri L, Tuchinda P. Factors predicting the response to cyclosporin treatment in patients with chronic spontaneous urticaria: a systematic review. Allergy Asthma Immunol Res. 2019;11:736-55.

26. Amber T, Tabassum S. Cyclosporin in dermatology: a practical compendium. Dermatol Ther. 2020;33:e13934.

27. Stanaland BE. Treatment of patients with chronic idiopathic urticaria. Clin Rev Allergy Immunol. 2002;23:233-40.

28. da Silva NL, Damayanthi H, Rajapakse AC, Rodrigo C, Rajapakse S. Lukotriene receptor antagonists for chronic urticaria: a systematic review. Allergy Asthma Clin Immunol. 2014;10:24.

29. Sarkar TK, Sil A, Pal S, Ghosh C, Das NK. Efectiveness and safety of levocetirizine $10 \mathrm{mg}$ versus a combination of levocetirizine $5 \mathrm{mg}$ and montelukast $10 \mathrm{mg}$ in chronic urticaria resistant to levocetirizine $5 \mathrm{mg}$ : A double-blind, randomized, controlled trial. Indian J Dermatol Venereol Leprol. 2017;83:561-8.
30. Akenroye AT, McEwan C, Saini SS. Montelukast reduces symptom severity and frequency in patients with angioedema-predominat chronic spontaneous urticaria. J Allergy Clin Immunol Pract. 2018;6:1403-5.

31. Haarman MG, van Hunsel F, de Vries TW. Adverse drug reactions of montelukast in children and adults. Pharmacol Res Per. 2017;5:e00341.

32. Iweala OI, Copenhaver C, Wu EY, Moran TP. Hydroxychloroquine as a steroid-sparing agente in an infant with chronic urticaria. Ann Allergy Asthma Immunol. 2018;120:102-4.

33. Boonpiyathad T, Sangasapaviliya A. Hydroxychloroquine in the treatment of anti-histamine refractory chronic spontaneous urticaria, randomized single-blinded placebo-controlled trial and an open label comparison study. Eur Ann Allergy Clin Immunol. 2017;49:220-4.

34. Seth S, Khan DA. The comparative safety of multiple alternative agentes in refractory chronic urticaria patients. J Allergy Clin Immunol Pract. 2017;5:165-70.e.2.

35. Antia C, Baquerizo K, Korman A, Alikhan A, Bernstein JA. Urticaria: A comprehensive review: treatment of chronic urticaria, special populations, and disease outcomes. J Am Acad Dermatol. 2018;79:617-3.

36. Morgan M, Khan DA. Therapeutic alternatives for chronic urticaria: an evidence-based review, part 1. Ann Allergy Asthma Immunol. 2008;100:403-12.

37. de Montojoye L, Herman A, Nicolas JF, Baeck M. Treatment of chronic spontaneous urticaria: immunomodulatory approaches. Clin Immunol. 2018;190:53-63.

38. Liang SE, Hoffmann R, Peterson E, Soter NA. Use of dapsone in the treatment of chronic idiopathic and autoimmune urticaria. JAMA Dermatology. 2019;155:90-5.

39. Morgan M, Cooke A, Rogers L, Adams-Huet B, Khan DA. Doubleblind placebo controlled trial of dapsone in antihistamine refractory idiopathic urticaria. J Allergy Clin Immunol. 2014;2:601-6.

40. Mehta A, Godse K, Patil S, Nadkarni N, Gautam M. Treatment of refractory chronic urticaria. Indian J Dermatol. 2015;60:230-7.

Não foram declarados conflitos de interesse associados à publicação deste artigo.

Correspondência:

Bruna Gehlen

E-mail: brugehlen@gmail.com 